OPEN ACCESS

Edited by:

Bert A 'T Hart,

University Medical Center Groningen,

Netherlands

Reviewed by:

Clara Ballerini,

Università Degli Studi di Firenze, Italy Andre Ortlieb Guerreiro Cacais, Karolinska Institute (KI), Sweden

*Correspondence:

Nathalie Arbour

nathalie.arbour@umontreal.ca

${ }^{\dagger}$ Present Address:

Sandrine L. Verstraeten, Louvain Drug Research Institute,

Cellular and Molecular Pharmacology, Brussels, Belgium

Specialty section:

This article was submitted to

Multiple Sclerosis and

Neuroimmunology,

a section of the journal

Frontiers in Immunology

Received: 03 October 2018

Accepted: 17 January 2019

Published: 06 February 2019

Citation:

Legroux L, Moratalla AC, Laurent C,

Deblois $G$, Verstraeten $S L$ and

Arbour N (2019) NKG2D and lts

Ligand MULT1 Contribute to Disease

Progression in a Mouse Model of

Multiple Sclerosis.

Front. Immunol. 10:154.

doi: 10.3389/fimmu.2019.00154

\section{NKG2D and Its Ligand MULT1 Contribute to Disease Progression in a Mouse Model of Multiple Sclerosis}

\author{
Laurine Legroux, Ana Carmena Moratalla, Cyril Laurent, Gabrielle Deblois, \\ Sandrine L. Verstraeten ${ }^{\dagger}$ and Nathalie Arbour*
}

Department of Neurosciences Université de Montréal, Montreal, QC, Canada

NKG2D is an activating receptor expressed on the surface of immune cells including subsets of T lymphocytes. NKG2D binds multiple ligands (NKG2DL) whose expression are differentially triggered in a cell type and stress specific manner. The NKG2D-NKG2DL interaction has been involved in autoimmune disorders but its role in animal models of multiple sclerosis (MS) remains incompletely resolved. Here we show that NKG2D and its ligand MULT1 contribute to the pathobiology of experimental autoimmune encephalomyelitis (EAE). MULT1 protein levels are increased in the central nervous system (CNS) at EAE disease peak; soluble MULT1 is elevated in the cerebrospinal fluid of both active and passive EAE. We establish that such soluble MULT1 enhances effector functions (e.g., IFN $\gamma$ production) of activated CD8T lymphocytes from wild type but not from NKG2D-deficient (KIrk $\left.1^{-/}\right)$mice in vitro. The adoptive transfer of activated $T$ lymphocytes from wild type donors induced a significantly reduced EAE disease in KIrk $1^{-1-}$ compared to wild type $\left(K I r k 1^{+/+}\right)$recipients. Characterization of $\mathrm{T}$ lymphocytes infiltrating the CNS of recipient mice shows that donor (CD45.1) rather than endogenous (CD45.2) CD4 T cells are the main producers of key cytokines (IFN $\gamma, \mathrm{GM}-\mathrm{CSF}$ ). In contrast, infiltrating CD8T Iymphocytes include mainly endogenous (CD45.2) cells exhibiting effector properties (NKG2D, granzyme B and IFN $\gamma$ ). Our data support the notion that endogenous CD8T cells contribute to passive EAE pathobiology in a NKG2D-dependent manner. Collectively, our results point to the deleterious role of NKG2D and its MULT1 in the pathobiology of a MS mouse model.

Keywords: T lymphocytes, central nervous system, autoimmune disease, neuroinflammation, NK cell lectin-like receptor subfamily

\section{INTRODUCTION}

Multiple sclerosis (MS) is an inflammatory disease of the central nervous system (CNS) characterized by demyelination, axonal loss, activation of glial cells, and infiltration of immune cells, such as macrophages and lymphocytes $(1,2)$. Although it is well established that the immune system participates in tissue destruction characteristic of MS, the contribution of specific immune mediators to the MS pathobiology has not been fully elucidated (2).

NKG2D is an activating or co-activating receptor expressed on several immune effector cells. Most human CD8 T lymphocytes and activated mouse CD8 T lymphocytes exhibit NKG2D on 
their surface as well as a small subpopulation of activated CD4 T lymphocytes in both species (3). NKG2D can recognize multiple ligands (NKG2DL); they include Rae-1 ( $\alpha, \beta, \gamma, \delta$, and $\varepsilon$ ), H60 $(a, b, c)$ and MULT1 in mice; whereas $\operatorname{ULBP}(1,2,3,4,5$, and 6) and MIC (A and B) are the human NKG2DL (4). The expression of NKG2DL are regulated at multiple levels (transcription, posttranscription, post-translation, etc.) and vary according to cell type, activation and environmental cues (5-7). Although weakly expressed under normal physiological conditions, NKG2DL are strongly induced in response to stress such as inflammation or infection $(4,6)$.

Shedding of NKG2DL by tumor cells has been reported in both humans and mice (8). Specific soluble murine and human NKG2DL can trigger NKG2D internalization on effector immune cells and consequently contribute to tumor immune evasion (8). In contrast, soluble MULT1, the murine NKG2DL exhibiting the highest affinity for NKG2D (9), can augment NK cell activation and increase tumor rejection (10). Moreover, soluble MICA, a human NKG2DL, can enhance NK cell functions in vitro (11). Soluble NKG2DL have been detected in the serum of patients affected by autoimmune diseases including MS (12-15); it is not fully understood, however, if and how these molecules impact autoimmune pathological processes.

Several studies have suggested that NKG2D and its ligands play a role in the pathobiology of MS. We have previously shown that multiple NKG2DL are detectable at the protein level on human oligodendrocytes in primary cultures (16). We demonstrated that disruption of the NKG2D-NKG2DL interaction inhibits killing of human oligodendrocytes mediated by activated human immune effectors including CD8 T lymphocytes (16). We detected oligodendrocytes expressing MICA/B in post-mortem MS tissues and CD8 T lymphocytes in close proximity to these MICA/B-expressing cells (16). Notably, Ruck and colleagues showed that CD4 T lymphocytes carrying NKG2D are enriched in the blood, cerebrospinal fluid and post-mortem brain lesions of MS patients compared to control donors especially during relapses (17). Whether NKG2D plays a role in MS pathobiology remains to be established.

Experimental autoimmune encephalomyelitis (EAE) is the most commonly used rodent model to investigate this neuroinflammatory disease as it recapitulates multiple immunopathological features of MS (18). Studies by different groups support the notion that NKG2D participates in EAE immunopathobiology. The group of Raulet assessed the susceptibility of NKG2D-deficient $\left(\mathrm{Klrk1}^{-/}\right)$mice to active EAE induction. They observed that using a suboptimal dose of the encephalitogenic myelin peptide $\mathrm{MOG}_{35-55}$, disease was slightly reduced in $\mathrm{Klrk1}^{-/-}$compared to wild type mice; such difference was not observed when optimal dose of MOG was injected (19). Wiendl's group showed that blocking NKG2D before, but not after, disease onset diminished active EAE disease severity and migration into the CNS of both CD4 and CD8 T lymphocytes (17). However, it remains unknown whether NKG2D acts via additional mechanisms in EAE pathobiology.
Here, we investigated the role of NKG2D and its ligands in the active and passive EAE models. We establish that one specific NKG2DL, MULT1, is significantly upregulated at the protein level in the spinal cord at the disease peak. Notably during EAE, the cleaved form of MULT1 is elevated not only in this organ but also in the cerebrospinal fluid. We show that soluble MULT1 increases the effector properties of CD8 T lymphocytes in vitro. Finally, we demonstrate using the adoptive model of EAE that endogenous $\mathrm{T}$ lymphocytes expressing NKG2D contribute to disease progression. Indeed, the adoptive transfer of wild type activated $\mathrm{T}$ lymphocytes triggers a significantly less severe disease and fewer CNS-infiltrated T lymphocytes in $\mathrm{Klrk1}^{-/-}$ compared to $K l r k 1^{+/+}$recipients. Overall, our results suggest that NKG2D and its ligands play a role in the pathobiology of MS mouse model.

\section{MATERIALS AND METHODS}

\section{Animals}

Wild type (WT) C57BL/6J (B6-CD45.2), B6.SJL-Ptprc ${ }^{a}$ $P e p c^{b} /$ BoyJ (B6-CD45.1), and Klrk1-/- (B6.Cg-Klrk1 $1^{\text {tm1Dhr} / J) ~}$ mice were obtained from The Jackson Laboratory (Bar Harbor, ME, USA). All mice were treated in strict adherence with approved protocols (N13043NAs and N17031NAs) from the CRCHUM Institutional Committee for the Protection of Animals and the Canadian Council on Animal Care.

\section{Experimental Autoimmune Encephalomyelitis (EAE) Disease Induction and Scoring}

Active EAE was induced as previously published (20). Briefly, 6-8 week old female mice were immunized subcutaneously with 200 $\mu \mathrm{g}$ of myelin oligodendrocyte glycoprotein 35-55 (MOG $\left.{ }_{35-55}\right)$ peptide emulsified in Complete Freund's adjuvant supplemented with $400 \mu \mathrm{g}$ of Mycobacterium tuberculosis (CFA-MOG $35-55$ ). Two days later, mice were intraperitoneally injected with $400 \mathrm{ng}$ of pertussis toxin (PTX).

For passive EAE, 6-8 week old female donor WT mice were similarly immunized with $\mathrm{CFA}-\mathrm{MOG}_{35-55}$ and injected intraperitoneally with $400 \mathrm{ng}$ of PTX. Eight days later, donor mice were sacrificed; lymph nodes and spleens were harvested and processed as described below. Cells were put in culture at $7 \mathrm{million} / \mathrm{ml}$ in complete RPMI $[10 \%(\mathrm{v} / \mathrm{v})$ of fetal bovine serum, $50 \mu \mathrm{M}$ of $\beta$-mercaptoethanol, $1 \mathrm{mM}$ of sodium pyruvate, $0.01 \mathrm{M}$ of HEPES, $1 \mathrm{X}$ non-essential amino acids solution, $2 \mathrm{mM}$ glutamine, $100 \mathrm{U} / \mathrm{ml}$ penicillin, and $100 \mu \mathrm{g} / \mathrm{ml}$ streptomycin] in the presence of $\mathrm{MOG}_{35-55}(20 \mathrm{ug} / \mathrm{ml})$, recombinant mouse IL-12 (10 ng/ml, R\&D Systems distributed by Cedarlane Laboratories Oakville, ON, Canada), recombinant human IL-2 (100 U/ml, Roche, Nutley, NJ) and mouse recombinant IL-15 (1 ng/ml, $\mathrm{R} \& \mathrm{D}$ Systems) pre-complexed (incubation $30 \mathrm{~min}$ at $37^{\circ} \mathrm{C}$ ) with recombinant mouse IL- $15 \mathrm{R} \alpha(4.67 \mathrm{ng} / \mathrm{ml}, \mathrm{R} \& \mathrm{D}$ Systems $)$ as published by others (21). After $72 \mathrm{~h}$ of culture, cells were washed, resuspended in Hank's Balanced Salt solution, filtered on $70 \mu \mathrm{m}$ 
cell strainer, counted and injected intraperitoneally into naïve $K l r k 1^{-/-}$and wild type $\left(K l r k 1^{+/+}\right)$female mice.

Passive and active EAE animals were scored according to the following scale: $0=$ normal, $1=\operatorname{limp}$ tail, $2=$ slow rightingreflex, 3 = paralysis of one hind limb, $3.5=$ paralysis of one hind limb and weakness in second hind limb, $4=$ paralysis of both hind limbs, $4.5=$ paralysis of both hind limbs and weakness in forelimbs, $5=$ moribund. Deeply anesthetized mice were perfused with $50 \mathrm{ml}$ of saline $0.9 \%(\mathrm{w} / \mathrm{v})$ prior to collect organs for RNA extraction, protein extraction, or organ processing for flow cytometry analysis.

\section{RNA Extraction and RT-qPCR}

Collected organs were put directly into TRIzol ${ }^{\circledR}$ Reagent (Life technologies Thermo Fisher Scientic, Burlington, ON, Canada). Total RNA was extracted from spleen, liver, spinal cord, brain stem-cerebellum and forebrain according to manufacturer's instructions. RNA samples were transcribed into cDNA using Quantitect Reverse transcription kit (Qiagen, Mississauga, $\mathrm{ON}$, Canada) as previously published (22). Gene expression levels were determined by quantitative real-time PCR using primers and TaqMan FAM-labeled probes from Applied Biosystems-ThermoFisher Scientific and the QuantStudio 7 Flex Real-Time PCR System. Amplification of mouse HPRT1 (Mm01545399_m1) was used as an endogenous control to assess the expression of murine NKG2D ligands: MULT1 (Mm01180648_m1), panRAE (Mm00558293_g1), H60b (Mm04243254_m1), and H60c (Mm04243526_m1).

\section{Protein Extraction}

Spinal cord, brain stem-cerebellum and forebrain were, respectively, homogenized in 500 ul of lysis buffer $[50 \mathrm{mM}$ Tris pH 7.5, $1 \mathrm{mM}$ EDTA, $150 \mathrm{mM} \mathrm{NaCl}, 1 \%$ (w/v) NP40, 1\% (w/v) SDS and cocktail of protease inhibitor containing $5 \mu \mathrm{g} / \mathrm{ml}$ of: Leupeptin (BioShop, Burlington, ON, Canada), Pepstatin A (BioShop) and Chymostatin (Sigma-Aldrich, Oakville, ON, Canada)]. Lysates were kept $10 \mathrm{~min}$ at room temperature before storing at $-80^{\circ} \mathrm{C}$.

\section{Cerebrospinal Fluid (CSF) Collection}

Deeply anesthetized mice were placed on the stereotaxic instrument with $135^{\circ}$ between head and body. Sagittal incision of the skin was made aligned with the ears, then the subcutaneous tissues and muscles were separated by blunt dissection and were hold by microretractor. The capillary tube was introduced into the cisterna magna though the dura mater, laterally to the arteria dorsalis spinalis. CSF was harvested into capillary tube, collected into Eppendorf tube and immediately frozen on dry ice. Samples were stored at $-80^{\circ} \mathrm{C}$.

\section{Western Blot}

Proteins isolated from organs were electrophoresed under reducing conditions and transferred onto PVDF membrane as previously published (23). CSF samples were run on a stain free gel (Bio-Rad Laboratories, Mississauga, ON, Canada) containing trihalo compounds reacting with tryptophan residues and used to revel total proteins on gel before transfer. MULT1 (rat monoclonal antibody clone 1D6 provided by Dr. S. Jonjic, University of Rijeka, Rijeka, Croatia) and Rae-1 (polyclonal goat anti-Rae-1 pan specific R \& D Systems), were quantified by chemiluminescence relative to total protein as published (24), actin (monoclonal antibody clone C4, MP Biomedicals, Fisher Scientific) or albumin (rabbit anti-albumin, Novus Biologicals, distributed by Cedarlane Laboratories) using the Chemidoc MP imaging system (Bio-Rad Laboratories).

\section{Immune Cell Isolation From CNS and Lymphoid Organs}

Brain and spinal cord were processed from individual mice as previously published (20). Briefly, minced CNS was digested 15 min with collagenase D and DNase I at $37^{\circ} \mathrm{C}$ prior to being mashed. Myelin was removed using Percoll. Harvested spleens and lymph nodes were put in complete RPMI and then mashed through cell strainer $(70 \mu \mathrm{m})$ to obtain single cell suspension. Red blood cells from spleens were lysed using $0.83 \%(\mathrm{w} / \mathrm{v})$ of ammonium chloride for $4 \mathrm{~min}$ at room temperature.

\section{Culture of Splenocytes}

Splenocytes from $K l r k 1^{+/+}$or $K l r k 1^{-/-}$mice were put in culture in complete RPMI in 48 well plates pre-coated with anti-CD3 antibody (2 ug/ml in $100 \mathrm{mM} \mathrm{NaHCO} 3$ overnight at $4^{\circ} \mathrm{C}, \mathrm{BD}$ Biosciences, Mississauga, ON, Canada), antiCD28 antibody (1 ug/ml BD Biosciences), recombinant human IL-2 $(100 \mathrm{U} / \mathrm{ml})$, and the complex recombinant mouse IL$15 / \mathrm{IL}-15 \mathrm{R} \alpha$ (described above). On day 5, recombinant murine MULT1 protein $(10 \mu \mathrm{g} / \mathrm{ml}, \mathrm{R} \& \mathrm{D}$ Systems $)$ was added or not to splenocytes. On day 7 , cells were activated for the detection of intracellular mediators and analyzed by flow cytometry as described below.

\section{Flow Cytometry}

Flow cytometry staining was performed as previously published $(22,25,26)$. Briefly, cells were incubated $15 \mathrm{~min}$ in blocking solution containing normal mouse immunoglobulins and rat anti-mouse CD16/CD32 prior to be incubated in the presence of fluorochrome-conjugated antibodies. Antibodies targeted CD45.1 (Biolegend, San Diego, CA, USA, clone A20), CD45.2 (BioLegend, distributed by Cedarlane Laboratories, clone 104), CD11b (BD Biosciences, clone M1/70), CD3 (BD Biosciences, clone 145-2C11), CD4 (BD Biosciences, clone RM4-5), CD8 (BD Biosciences, clone 53-6.7), and NKG2D (eBioscience or R\&D systems, respectively, clone CX5 or 191004). LIVE/DEAD Fixable Aqua Dead Cell Stain was used to exclude dead cells. To trace proliferation of MOG-specific T cells, leukocytes from MOGimmunized donor mice were labeled with CFSE as previously done $(22,25)$ prior to being activated in vitro for $72 \mathrm{~h}$. For cytokine detection, cells were stimulated $5 \mathrm{~h}$ with phorbol 12 myristate 13 -acetate $(20 \mathrm{ng} / \mathrm{ml}$, Sigma-Aldrich) and ionomycin (1 ug/ml, Sigma-Aldrich) in the presence of brefeldin A (5 $\mathrm{ug} / \mathrm{ml}$, Sigma-Aldrich) and monensin sodium (1 $\mu \mathrm{M}$ SigmaAdrich). Intracellular staining was accomplished as previously published (25). Antibodies targeted interferon- $\gamma$ (IFN $\gamma$, BD Biosciences clone MP6-XT22), granulocyte-macrophage colonystimulating factor (GM-CSF, BD Biosciences, clone MP1-22E9), 
interleukin-17 (IL-17, BD Biosciences, cloneTC11-18H10) and granzyme B (eBioscience ThermoFisher Scientific, clone 16G6). Appropriate isotype controls were used in all steps. Staining specificity was confirmed using fluorescence minus one (FMO: all antibodies minus one). The median fluorescence intensity (MFI) was calculated by subtracting the fluorescence of the isotype from that of the stain. Cell numbers were quantified using either cell counting prior to cytometry staining or beads added to samples prior to sample acquisition as previously described (20).

\section{Immunohistochemistry}

Deeply anesthetized mice were perfused with $30 \mathrm{ml}$ of saline $0.9 \%(\mathrm{w} / \mathrm{v})$ and then with $50 \mathrm{ml}$ of paraformaldhehyde $4 \%$ (w/v in PBS). Spinal cord was collected and soaked into $4 \%$ paraformaldehyde for 1 day prior to being transferred into sucrose $30 \%(\mathrm{w} / \mathrm{v})$ for 2 days and then put into OCT for freezing at $-80^{\circ} \mathrm{C}$. Nine micron sections were stained for FluoroMyelin ${ }^{\mathrm{TM}}$ Green fluorescent myelin stain (Thermofisher Scientific) and 2(4-Amidinophenyl)-6-indolecarbamidine dihydrochloride, 4',6Diamidino-2-phenylindole dihydrochloride (DAPI) (SigmaAldrich) for nucleus detection according to manufacturers' instructions. Slides were observed using a SP5 Leica confocal microscope and confocal images acquired sequentially in different channels using LASAF software and overlay using Adobe Photoshop software.

\section{Statistical Analysis}

Data analysis was performed using Prism 7.0 software (GraphPad, La Jolla, CA, USA). Statistical tests used are indicated in figure legends. Values were considered statistically significant when probability $(\mathrm{P})$ values were equal or below 0.05 $\left({ }^{*}\right), 0.01\left(^{* *}\right)$, or $0.001\left(^{* * *}\right)$.

\section{RESULTS}

\section{NKG2D has a Modest Impact on Active EAE}

To assess the impact of NKG2D on the development of EAE, we applied our standard protocol on mice lacking NKG2D $\left(K l r k 1^{-/-}\right)$and wild type $\left(K l r k 1^{+/+}\right)$counterparts. We observed a modest and transient (day 11 to 14 ) enhanced peak of disease in $\mathrm{Klrk1}^{-/-}$compared to wild type mice when large groups of animals were analyzed $(n=22-25)$ (Figure 1A). We observed similar proportions of infiltrating CD4 $(67-72 \%$ of total CD3 T lymphocytes) and CD8 (14-17\% of total CD3 T lymphocytes) $\mathrm{T}$ cells in the CNS of both groups (Figures 1B,C). Within these CNS-infiltrated $\mathrm{T}$ lymphocytes, only a very small proportion of CD4 T lymphocytes expressed NKG2D (less than 8\%) whereas $28 \%$ of CD8 T lymphocytes carried detectable levels of this receptor in $K l r k 1^{+/+}$mice (Figure 1D). As expected, a negligible proportion of T lymphocytes expressed NKG2D in the Klrk1-/mice (Figures 1B,D). The total number of T cells, CD4 and CD8 $\mathrm{T}$ cells that infiltrated the CNS during EAE were similar in both genotypes (Figure 1E). Similar to what others published (19), NKG2D had a minimal impact on the neurobehavioral score of EAE mice. Our results show that during EAE, amongst the CNS-infiltrated $\mathrm{T}$ lymphocytes, a greater proportion of
CD8 than CD4 T lymphocytes carried NKG2D but as CD4 T cells were more abundant, NKG2D-expressing CD4 and CD8 T lymphocytes were present in similar number per CNS in wild type mice (Figure 1E).

\section{EAE Induction Modestly Alters the Expression of Rae-1 and $\mathrm{H} 60$ Ligands}

As each NKG2DL can be induced in response to different triggers and exhibits distinct biological functions, we assessed the expression of the specific ligands (H60 (b-c), Rae1 $(\alpha-\varepsilon)$ and MULT1) known to be transcribed in C57BL/6 mice $(9,27-$ 29), during the development of EAE. To investigate whether the presence of NKG2D-expressing immune effectors affects the ligands' expression, we quantified the relative mRNA levels of NKG2DL in $K l r k 1^{-/-}$and wild type $\left(K l r k 1^{+/+}\right)$mice at the different EAE stages: pre-symptomatic, onset and peak stages. We analyzed three CNS areas: spinal cord, brain stem/cerebellum and forebrain and observed that the mRNA levels of H60b did not significantly vary in the wild type mice (Figure 2A). We detected a slight but significant decrease in the spinal cord of $\mathrm{Klrk1}^{-/-}$ mice compared to controls at disease onset and a slight increase in the brainstem-cerebellum (Figure 2A). H60c mRNA was not detected in all tested organs (CNS, spleen, etc.) except the eye (data not shown). Using a pan-Rae-1 qRT-PCR assay to detect all forms of Rae-1, we observed a small but significant increase at the peak of disease in wild type mice in the spinal cord compared to controls as previously reported by others (30). However, Rae1 mRNA levels were decreased in the spinal cord of $K l r k 1^{-/-}$ mice at the presymptomatic and onset stages as well as in the forebrain at the presymptomatic stage (Figure 2A). Notably, the levels in $\mathrm{Klrk1}^{-/-}$mice were significantly lower than in $K l r k 1^{+/+}$ at the same disease stage for the presymptomatic and onset in the spinal cord and the brainstem-cerebellum (Figure 2A). We also assessed the protein expression of Rae-1 in these CNS areas during the development of EAE. The relative amounts of Rae-1 did not vary between control mice (CFA without MOG) and EAE animals in all three CNS areas tested (spinal cord, brainstem/cerebellum, forebrain) (Figures 2B,C).

\section{Enhanced Expression of MULT1 in the CNS of EAE Mice}

In contrast, MULT1 mRNA levels were significantly enhanced in the spinal cord of both $K l r k 1^{+/+}$and $K l r k 1^{-/-}$groups at the peak of EAE compared to controls, presymptomatic and onset time points (Figure 3A). Upregulated MULT1 mRNA levels during EAE were previously reported by others (30). Moreover, MULT1 mRNA levels were greater in $K l r k 1^{-/-}$mice compared to wild type counterparts (Figure 3A) without reaching statistical significance. These observations suggest that in the presence of NKG2D-expressing immune effectors, MULT1-expressing cells may down-regulate such expression or may be destroyed. In the brainstem-cerebellum and forebrain, we also detected significantly augmented MULT1 mRNA levels at the peak of EAE compared with other disease stages in both $K l r k 1^{+/+}$or $K l r k 1^{-/-}$ mice (Figure 3A). We observed a small increase of MULT1 mRNA levels in the spleen of $K l r k 1^{-/-}$and $K l r k 1^{+/+}$mice at 


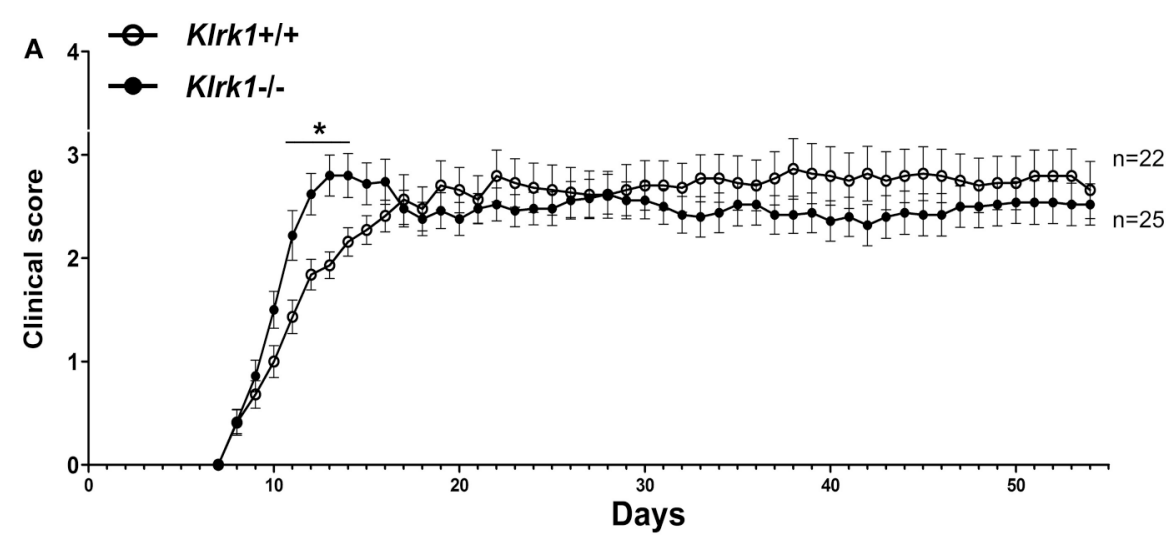

B
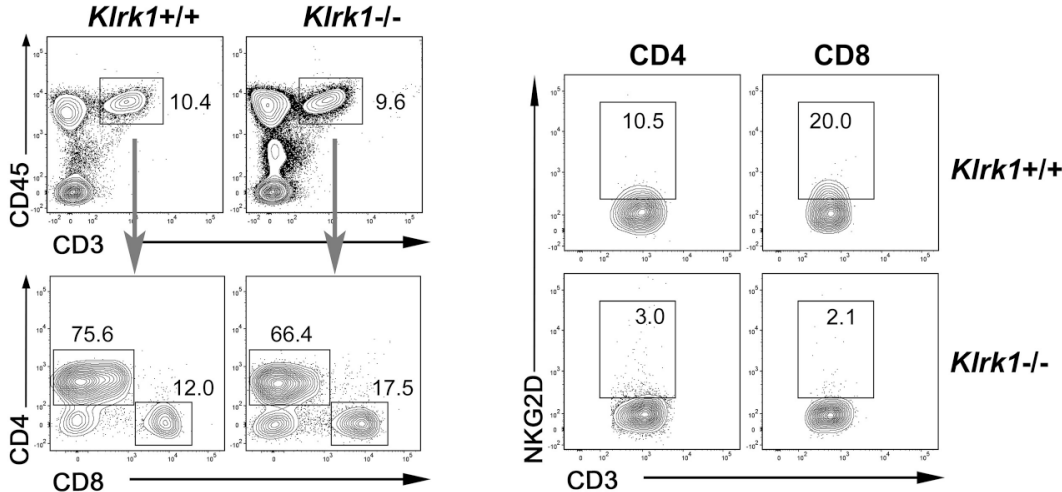

C

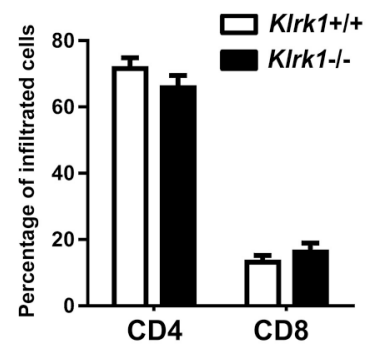

D

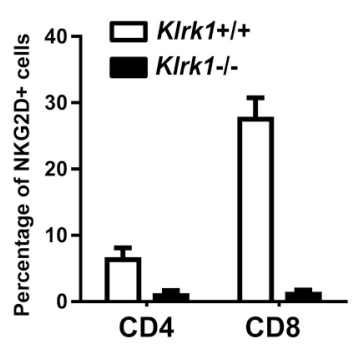

E

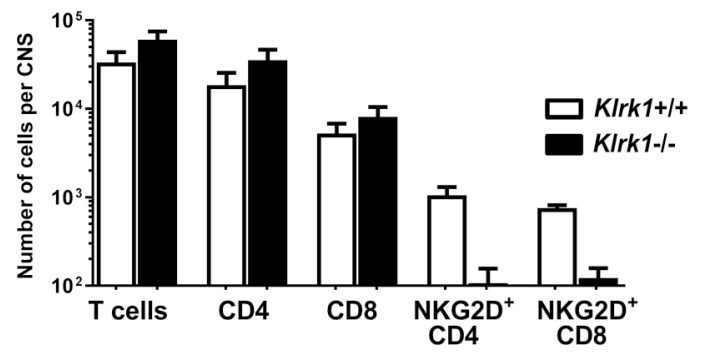

FIGURE 1 | Active EAE disease course is similar in the absence of NKG2D. Active EAE was induced in KIrk $1^{-/-}$(black circles) and wild type (KIrk $1^{+/+}$, open circles) mice. Clinical score was assessed over 55 days or CNS infiltrating T lymphocytes characterized by flow cytometry. (A) Clinical score of $K / r k 1^{+/+}(n=22)$ and KIrk1 $1^{-/-}$mice $(n=25)$ presented as mean \pm SEM. 2way ANOVA KIrk $1^{-/-}$vs. KIrk $1^{+/+}$day 11 to $14{ }^{*} p<0.05$. (B) Representative contour plots show gating for T cells $\left(\mathrm{CD} 45^{\mathrm{hi}} \mathrm{CD}^{+}\right)$and then CD4 and CD8 as well as the detection of NKG2D on CD4 and CD8T cell subsets from CNS cells of Klrk $1^{-/-}$and Klrk $1^{+/+}$mice. (C) Percentage of CNS infiltrated CD4 and CD8T lymphocytes within CD3T cells. Mean \pm SEM $n=3$. (D) Percentage of CNS-infiltrated CD4 and CD8T lymphocytes expressing NKG2D. Mean $\pm \mathrm{SEM} n=3$. (E) Number of cells per CNS (spinal cord and brain pooled from each individual mouse) for T cells $\left(C D 45^{\text {hi }} \mathrm{CD} 3^{+}\right.$), CD4 and CD8T cells as well as NKG2D ${ }^{+}$CD4 T cells and NKG2D ${ }^{+}$CD8T cells. Mean $\pm \mathrm{SEM} n=3$. 

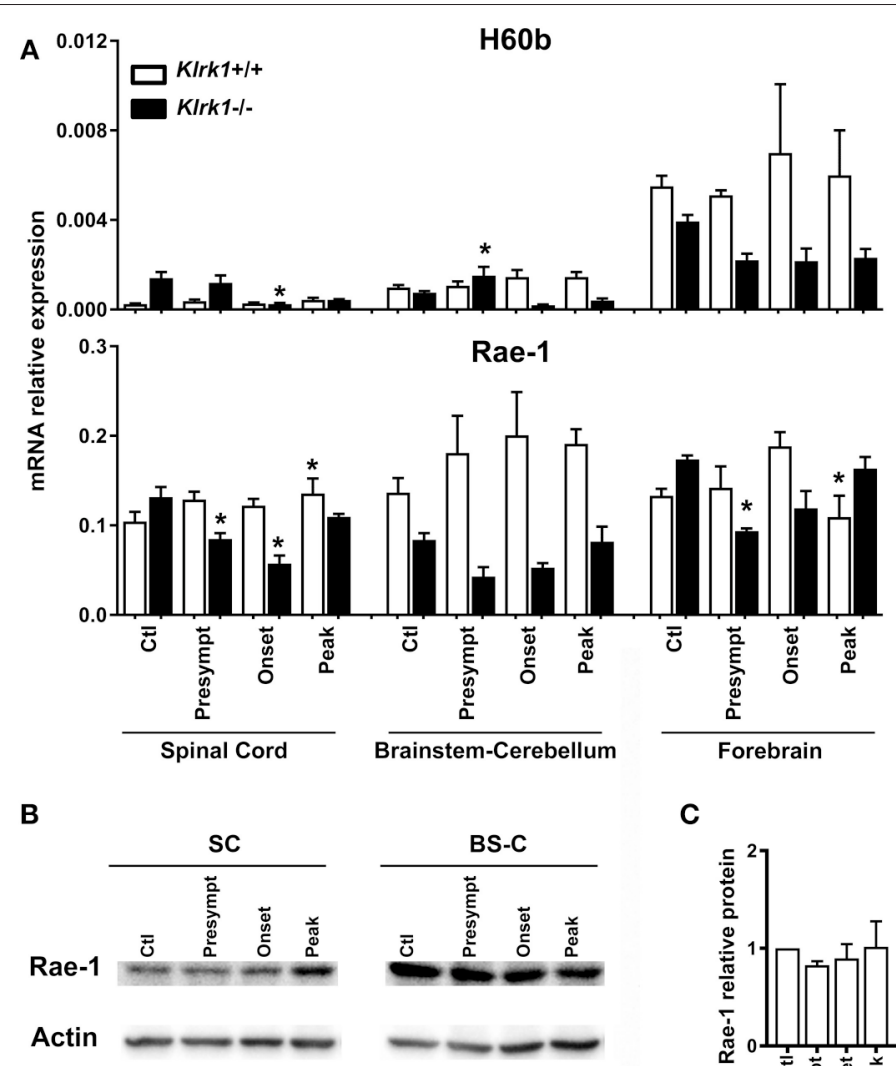

C

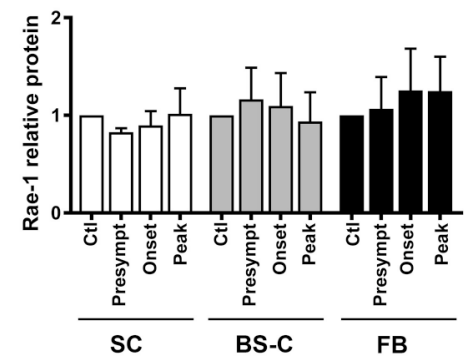

FIGURE 2 | Variations in CNS expression of Rae-1 only at the mRNA level. (A) Relative mRNA expression of H60b and Rae-1 in CNS areas [spinal cord (SC), brainstem-cerebellum (Bs-C) and forebrain (Fb)] from Klrk $1^{-/-}$(black bars) and wild type (KIrk $1^{+/+}$; white bars) mice either injected with CFA-PBS as control (Ctl) or subjected to active EAE and sacrificed at pre-symptomatic (presympt), onset or peak stage of disease. Kruskal-Wallis test and Dunn's test analysis within the same genotype group. Statistical differences for H6Ob in KIrk1-/- group: spinal cord onset vs. control or presymptomatic; brainstem-cerebellum presymptomatic vs. onset or peak. Statistical differences for Rae-1 expression in KIrk1+/+ group: spinal cord: peak vs. ctl; forebrain onset vs. peak. Statistical differences for Rae-1 expression in KIrk $1^{-/-}$group: spinal cord control vs. presymptomatic or onset; for forebrain presymptomatic vs. control or peak. * $<<0.05$. (B,C) Western blot analysis of Rae-1 expression in the spinal cord (SC, brainstem-cerebellum (Bs-C) and forebrain (Fb) from wild type mice $\left(K / r k 1^{+/+}\right.$) either injected with CFA-PBS as control (Ctl) or subjected to active EAE and sacrificed at pre-symptomatic (presympt), onset or peak stage of disease. (B) One representative Western blot and (C) Rae-1 relative expression to actin, ctl for each organ defined as 1 . Mean $\pm \operatorname{SEM} n=5$.

disease peak and no variation in the liver (Figure 3B). These results demonstrate that during active EAE, MULT1 expression is significantly upregulated (over $900 \%$ increase in spinal cord) in the CNS.

To determine whether MULT1 expression is also increased at the protein level, we performed western blot analysis on the same CNS areas. We observed two bands representing the full length (50-55 kDa) and the putative cleaved extracellular domain $(25 \mathrm{kDa})$ of MULT1 at the peak of disease, which was absent in controls mice (Figure 3C). The quantification of MULT1 relative to actin showed that the spinal cord contained greater levels of both MULT1 forms (55 and $25 \mathrm{kDa}$ ) compared to brainstem/cerebellum and forebrain areas in $K l r k 1^{+/+}$as well as $K l r k 1^{-1-}$ mice at disease peak (Figure 3D). The full and cleaved forms of MULT1 were detected in the spinal cord at the onset of EAE only in a subset of mice (4 out of 12 mice). Notably, greater amounts of the $25 \mathrm{kDa}$ band relative to the $55 \mathrm{kDa}$ band at disease peak were observed in both $K l r k 1^{+/+}$and $K l r k 1^{-/-}$ mice (Figures 3E,F). Our results strongly suggest that MULT1 is the NKG2DL with the greatest enhanced mRNA and protein expression levels at the peak of EAE in the CNS, especially in the spinal cord, regardless of the presence of NKG2D-expressing immune cells.

\section{Elevated Expression of Soluble MULT1 in the CSF of EAE Mice}

To establish whether soluble MULT1 diffuses in the CNS, we performed western blots on CSF collected from control or EAE mice at different stages (pre-symptomatic, onset, peak) in $K l r k 1^{+/+}$and $K l r k 1^{-/-}$mice (Figure 4A). We observed that both forms (25 and $55 \mathrm{kDa}$ ) of MULT1 were detectable in the CSF and those levels were greater at disease peak compared to 


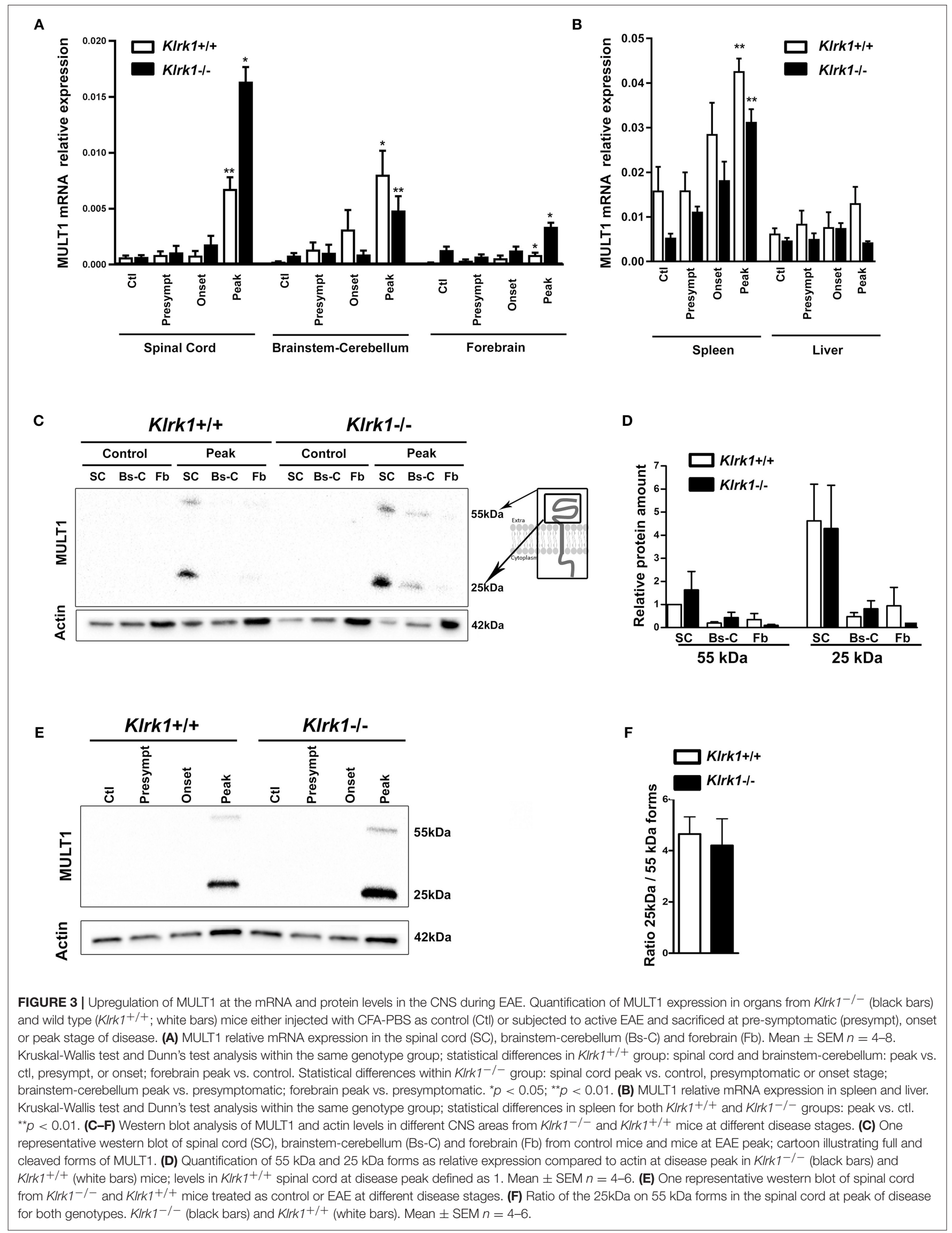



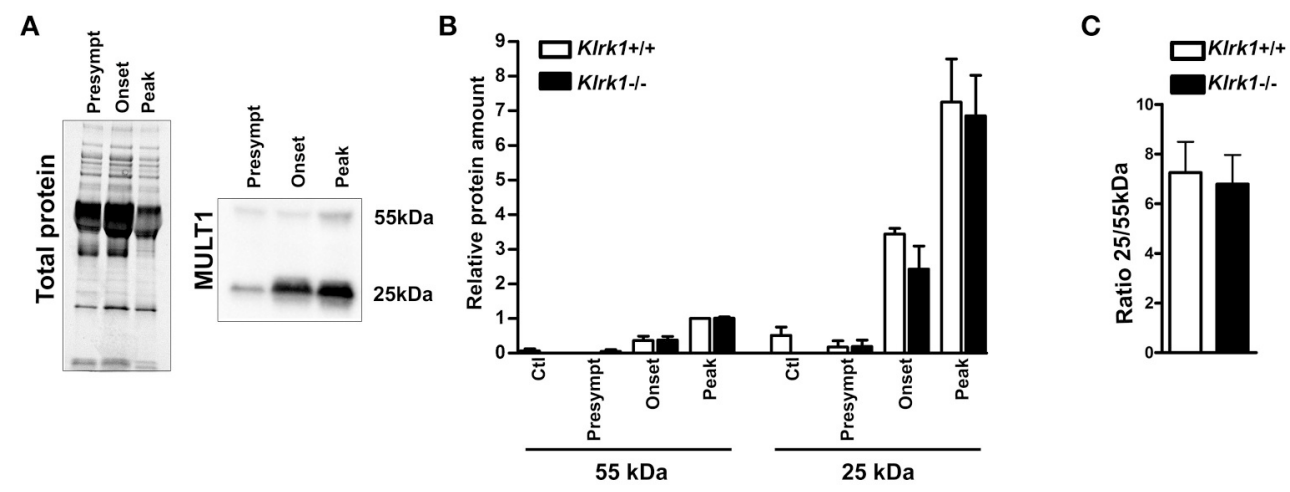

FIGURE 4 | Increased levels of soluble MULT1 in the CSF during EAE. Quantification of soluble MULT1 in CSF from KIrk1-/- (black bars) and wild type counterparts $\left(K I r k 1^{+/+}\right.$; white bars) either injected with CFA-PBS as control (Ctl) or subjected to active EAE and sacrificed at pre-symptomatic (presympt), onset or peak stage of the disease. (A) One representative western blot for the detection of total protein and MULT1. (B) Quantification of $55 \mathrm{kDa}$ and $25 \mathrm{kDa}$ forms as relative expression compared to total protein; levels in Klrk $1+/+$ at disease peak defined as 1 . Mean $\pm \mathrm{SEM} n=3-4$. (C) Ratio of the $25 \mathrm{kDa} / 55 \mathrm{kDa}$ forms at disease peak. Mean \pm $\operatorname{SEM} n=4$.

other EAE stages (Figure 4B). Notably, the $25 \mathrm{kDa}$ form was 7 fold more abundant than the $55 \mathrm{kDa}$ form in the CSF (Figure 4C) compared to 4.5 -fold increased expression in the spinal cord protein homogenates at the same disease stage (Figure 3F).

\section{Soluble MULT1 Released During EAE Enhances T Cell Responses}

We next sought to determine whether soluble MULT1 could impact activated $\mathrm{T}$ lymphocytes, which are abundantly present in the CNS of EAE mice. Splenocytes from naïve $K l r k 1^{+/+}$ and $K l r k 1^{-/-}$mice were activated in vitro with anti-CD3+antiCD28+IL-2+IL-15 to enhance NKG2D expression on CD8 T lymphocytes to similar levels observed on CNS-infiltrating CD8 T cells. After 5 days, recombinant mouse MULT1 or PBS was added to these activated cultures and 2 days later, cells were evaluated for their production of mediators by flow cytometry. Recombinant MULT1 was obtained from the same commercial source than what has been used by the Raulet's group to perform in vitro experiments with activated murine immune cells (10). Our in vitro culture conditions gave rise to a similar proportion $(37 \%)$ of activated $K l r k 1^{+/+}$ CD8 T lymphocytes expressing NKG2D (Figures 5A,B) as we observed on CNS-infiltrating CD8 T lymphocytes in EAE mice (Figure 1C, 28\%). As expected, NKG2D expression was negligible on $\mathrm{Klrk1}^{-/-}$cells. Notably, the addition of soluble MULT1 did not alter the percentage of cells expressing NKG2D; but slightly reduced NKG2D expression levels per cell in $K l r k 1^{+/+}$CD8 T cells [Figure 5C; MFI for NKG2D on Klrk1 $1^{+/+}$ CD8 T cells exposed to PBS (1279) vs. MULT1 (1068)]. The addition of MULT1 increased the percentage of granzyme B-expressing CD8 T lymphocytes (Figure 5B; \% granzymeexpressing Klrk1 $1^{+/+}$CD8 T cells exposed to PBS (44\%) vs. MULT1 $(67 \%) p=0.09)$; these cells expressed greater levels of this lytic enzyme compared to cells not treated with MULT1 (Figure 5C; granzyme B-MFI in $K l r k 1^{+/+}$CD8 $\mathrm{T}$ cells exposed to PBS (3094) vs. MULT1 (4854) $p=0.1$ ). MULT1 did not alter the percentage of IFN $\gamma$ producing $K l r k 1^{+/+} \mathrm{CD} 8 \mathrm{~T}$ lymphocytes $(80-86 \%)$ but significantly increased their cellular expression level (Figures 5A,C; IFN $\gamma$-MFI on $K l r k 1^{+/+}$CD8 T cells exposed to PBS (3095) vs. MULT1 (4901) * $p=0.0133)$. The enhancing impact of MULT1 on CD8 T cell effector functions was dependent on the presence of NKG2D as this soluble ligand did not alter the production of granzyme B or IFN $\gamma$ by $K l r k 1^{-/-}$ CD8 T lymphocytes as similar percentage of cells and MFI were observed for these cells in the PBS and MULT1 conditions (Figure 5). Our results suggest a novel activating role of soluble MULT1 on CD8 T lymphocytes.

\section{NKG2D Significantly Augments Passive EAE Severity}

Active EAE is strongly mediated by CD4 T lymphocytes but only a small proportion of these lymphocytes can express NKG2D upon activation. In contrast, a great proportion of activated murine CD8 T cells acquire NKG2D. To investigate the contribution of this receptor, we optimized a passive EAE model in which the transferred $\mathrm{T}$ cells include a similar proportion of CD8 and CD4 T cells. Lymphocytes from MOG-immunized donor mice were activated in vitro in the presence of IL-2, IL-12, and IL-15 prior to their transfer into naïve recipients. The addition of this cytokine cocktail led to the expansion of similar proportions of CD4 and CD8 T cells (data not shown). Activated cells were transferred into naive $K l r k 1^{+/+}$ and $K l r k 1^{-/-}$mice and clinical score recorded for 2 months (Figure 6A). Disease onset occurred similarly around day 10 post-transfer in both mouse groups (Figure 6A). However, Klrk $1^{+/+}$recipients achieved a significantly more elevated clinical score than the $K l r k 1^{-/}$recipients (Figure 6A). The dampened disease in $K l r k 1^{-/-}$recipients was maintained for the whole observation window. As these mice were injected in parallel with the same activated lymphocytes, the differences we observed could not be due to the transferred cells. Our results suggest that in passive EAE, endogenous immune cells contribute 


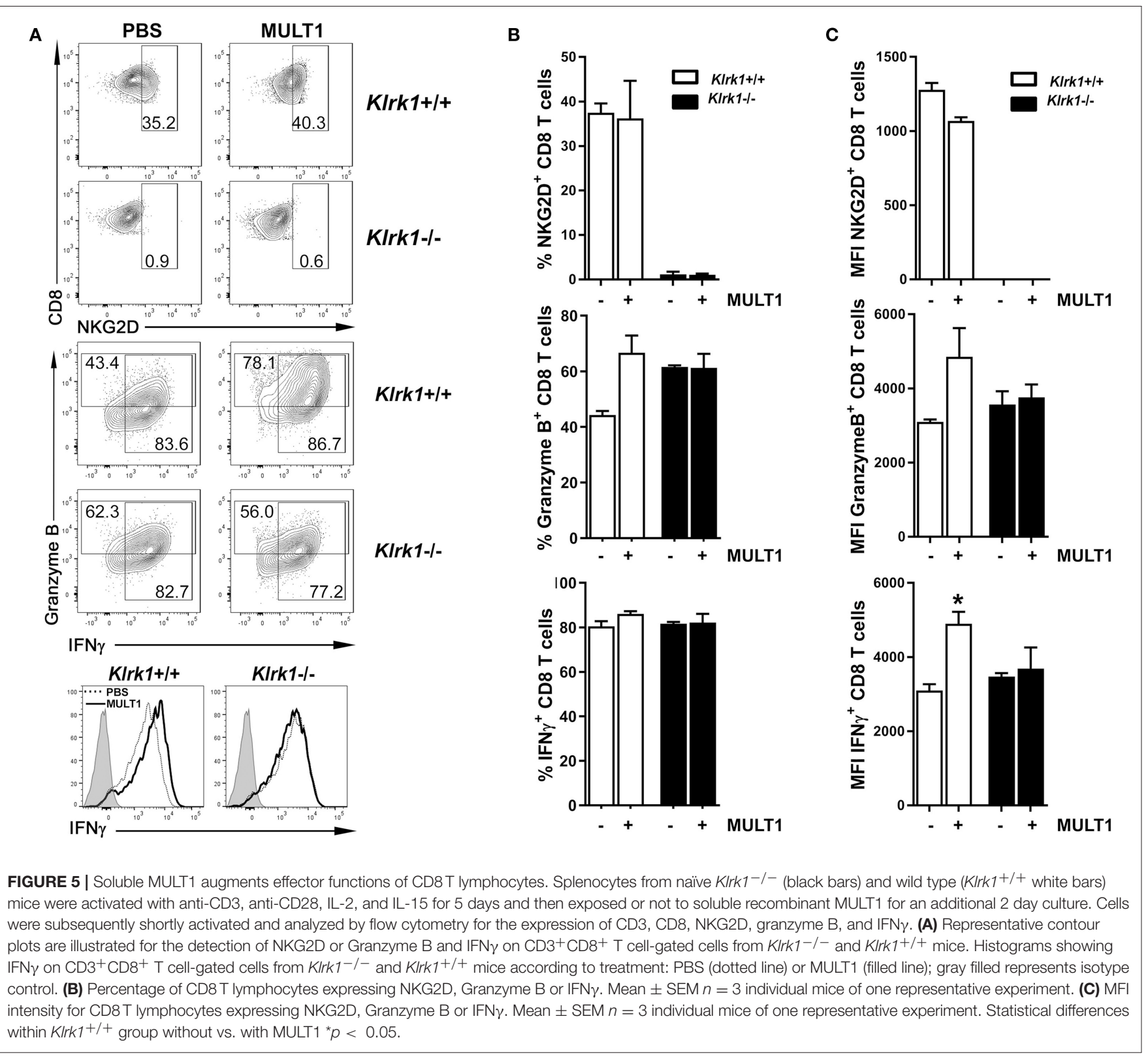

to disease pathobiology and that such impact depends on the endogenous expression of NKG2D.

We evaluated whether the injected cells contain MOG-specific $\mathrm{T}$ cells. Lymph node leukocytes from MOG-immunized donor mice were labeled with CFSE and activated in vitro following our usual protocol in the presence of MOG. Control cells were cultured in the same conditions but in the absence of the myelin peptide. We observed that our activation protocol efficiently induced proliferation as assessed by CFSE dilution (Figure 6B). Moreover, the proportion of CD4 and CD8 T lymphocytes that divided during the in vitro expansion and could produce key immune effectors (GM-CSF, IFN $\gamma$, IL-17 for CD4; GM-CSF, IFN $\gamma$, and Granzyme B for CD8) was greater in the presence of MOG (Figure 6B). We suggest that in vitro MOG addition had a more modest impact on CD4 than CD8 T lymphocytes due to the greater efficiency of antigen presenting cells activated upon immunization at activating CD4 than CD8 T lymphocytes. These results support the idea that at least a subset of transferred $\mathrm{T}$ lymphocytes was MOG-specific. In addition, the passive transfer of MOG-activated lymphocytes caused demyelination in recipient mice. Indeed, fluoromyelin staining revealed zones of demyelination (white arrows Figure 6C) in both $K l r k 1^{+/+}$ and $K l r k 1^{-/-}$mice. Finally, we also detected soluble MULT1 in the CSF of passive EAE mice supporting the notion that this NKG2DL is also elevated in this form of disease (Figure 6D). Notably, the relative amount of the $25 \mathrm{kDa}$ form of MULT1 was significantly more elevated in CSF from $K l r k 1^{+/+}$compared to Klrk1 ${ }^{-/-}$(Figure 6D). 

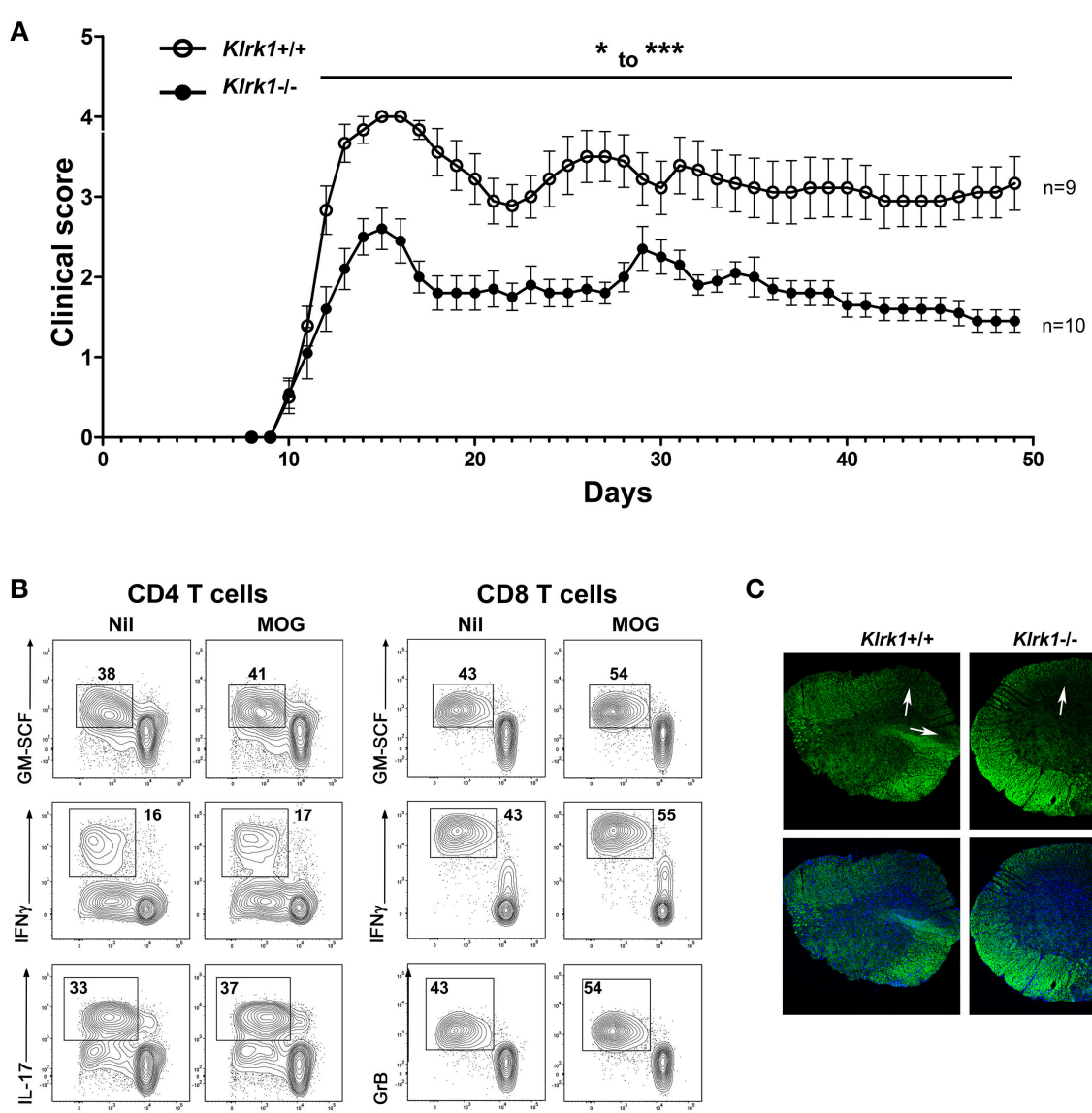

C

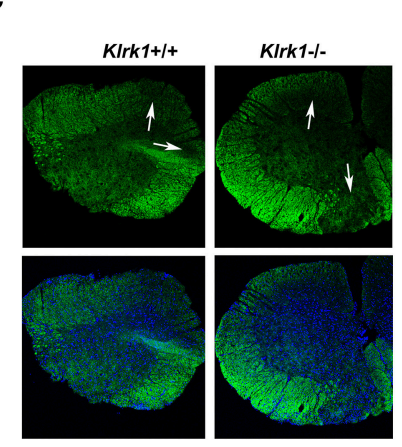

CFSE

D
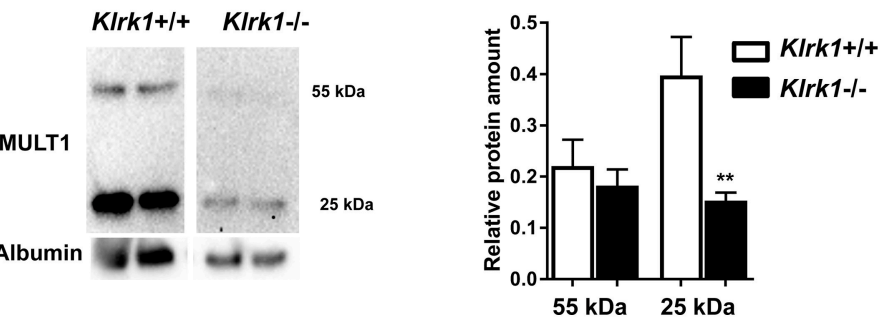

FIGURE 6 | Passive EAE is less severe in KIrk1 ${ }^{-/-}$than in wild type recipients. Leukocytes from MOG-immunized C57BL/6 were reactivated in vitro and then adoptively transferred to KIrk $1^{-/-}$(black) and wild type (KIrk $1^{+/+}$; white) recipients. (A) Mice were followed for clinical score. Mean \pm SEM, $n=9-10.2$ way ANOVA KIrk $1^{-/-}$vs. Klrk $1^{+/+}$day $22-23{ }^{*} p<0.05$ for all other days from day 12 to the end: ${ }^{* \star *} p<0.001$. (B) Flow cytometry analysis of ex vivo expanded T lymphocytes from MOG-immunized donor mice. Lymph node cells were collected, labeled with CFSE, and put in culture as described in materials and methods in the absence or presence of $\mathrm{MOG}_{35-55}$ for $72 \mathrm{~h}$. PMA, ionomycin, brefeldin $\mathrm{A}$ and monensin were added for $4 \mathrm{~h}$ prior to flow cytometry staining and analysis for intracellular mediators: GM-CSF, IFN $\gamma$, IL-17 and Granzyme B (GrB) as indicated. Contour plots illustrate gated events on CD4 or CD8 T cells expanded in vitro in the absence (Nil) or presence of MOG. (C) Representative detection of demyelination using fluoromyelin and DAPI in spinal cord sections from KIrk $1^{-/-}$and $K I r k 1^{+/+}$mice. White arrows indicate zones of myelin loss. (D) Western blot detection of MULT1 and albumin in CSF from KIrk $1^{-/-}$and $K I r k 1^{+/+}$mice 50 days after the adoptive transfer of activated lymphocytes. One representative western blot is illustrated and quantification of $55 \mathrm{kDa}$ and $25 \mathrm{kDa}$ forms as relative expression compared to albumin Mean $\pm \mathrm{SEM} n=7$. Statistical differences for relative abundance of MULT1 25KDa form KIrk1+/+ vs. KIrk1-/-** $p<0.01$.

\section{CNS-Infiltrated Endogenous CD8T Cells Exhibit Effector Functions}

To investigate the contribution of endogenous vs. transferred $\mathrm{T}$ cells, we used C57BL/6 mice expressing the pan leukocyte marker CD45.1 as donor mice for the transfer into the CD45.2-expressing $K l r k 1^{+/+}$and $K l r k 1^{-/-}$recipients. Mice were sacrificed 25 days post-transfer and CNS cells were analyzed by flow cytometry. We could easily discriminate donor $\left(\mathrm{CD} 45.1^{+}\right)$ from recipient immune cells $\left(\mathrm{CD} 45.2^{+}\right)$(Figure 7A). CD $45^{\text {int }}$ $\mathrm{CD}_{11 \mathrm{~b}}{ }^{+}$microglia all expressed the endogenous CD45.2 allele (data no shown). We compared CD45.2 hi+ immune cells to the CD $45.1^{+}$cells in $K l r k 1^{+/+}$and $K l r k 1^{-/-}$recipients. Most 
transferred cells $\left(\mathrm{CD} 45.1^{+}\right)$detected in the CNS were either CD4 or CD8 T cells (Figure 7B). Despite the injection of similar numbers of CD45.1 ${ }^{+}$CD4 and CD8 T cells, donor CD8 T cells represented a very small proportion of CNS-infiltrated $\mathrm{T}$ cells, especially in $\mathrm{Klrk1}^{-/-}$mice, while donor CD4 T cells were well represented. In contrast, amongst the endogenous immune cells present within the CNS, a greater proportion were CD8 T cells (41 \% in $K l r k 1^{+/+}$and $44 \%$ in $K l r k 1^{-/-}$) compared to CD4 T cells (30.7\% and $28.8 \%$ ) (Figure 7 B). The spleens of all animals contained very low proportion of CD $45.1^{+}$(donor) $\mathrm{T}$ cells (around 1\%) suggesting that the transferred cells either died or migrated to other sites such as the CNS at the time point tested (over 20 days after transfer). Overall, less transferred T cells were detected in the CNS of $K l r k 1^{-/-}$recipients than wild type counterparts correlating with the reduced disease severity in Klrk $1^{-/-}$recipients (Figure $7 \mathbf{B}$ ).

To characterize the properties of CNS-infiltrated $\mathrm{T}$ cells, we assessed the production of key immune effector molecules: IFN $\gamma, \mathrm{GM}-\mathrm{CSF}$ and granzyme B in CD45.1 ${ }^{+}$or CD45.1-negative $\left(\mathrm{CD} 45.2^{+}\right) \mathrm{CD} 4$ and CD8 T cells in both $K l r k 1^{+/+}$and $K l r k 1^{-/-}$ recipients. Production of IFN $\gamma$ by CNS-infiltrated CD4 and CD8 T cells was easily detected in both mouse groups. Notably, a significantly lower proportion of endogenous (CD45.2) CD4 T cells produced this cytokine compared to the counterparts originating from the transfer $\left({ }^{*} p<0.05\right)$ in $K l r k 1^{+/+}$mice (Figure 7C). In contrast, the proportion of endogenous (CD45.2) CD8 $\mathrm{T}$ cells producing IFN $\gamma$ was significantly higher than within transferred CD8 T cells $\left({ }^{* *} p<0.01\right.$; Figure 7D). A relatively small but similar proportion of endogenous and transferred CD4T cells expressed granzyme B (Figure 7C). However, the percentage of CD8 T cells carrying this lytic enzyme was significantly greater in the endogenous cells than in the transferred one $(p<0.05$, Figure 7D). We observed a significantly greater proportion of transferred (CD45.1) than endogenous (CD45.2) CD4 T cells producing GM-CSF (Figure 7C). Only few or no CD8 T cells produced GM-CSF or IL-17 above background level (data not shown). Notably, elevated proportions of endogenous CD4 and CD8 T cells in the CNS of $\mathrm{Klrk1}^{-/}$recipients produced the immune mediators tested (IFN $\gamma, \mathrm{GM}-\mathrm{CSF}$ or granzyme B).

We also measured the expression of NKG2D and could observe that as expected none of the endogenous CD8 T cells carry this receptor in the $K l r k 1^{-/-}$recipients. Nevertheless, a significantly higher percentage of endogenous (CD45.2) than transferred (CD45.1) CD8 T cells exhibited NKG2D in wild type recipients. It was not possible to compare transferred and endogenous $\mathrm{CD} 8 \mathrm{~T}$ cells in $K l r k 1^{-/-}$recipients due to the absence or very low number of CD45.1 ${ }^{+}$CD8 $\mathrm{T}$ cells in these mice (Figure 7D). A very low percentage of CD4 T cells expressed NKG2D in our model precluding any comparison. Our results suggest that both endogenous and transferred CD8 T cells could recognize NKG2D ligands in the CNS during passive EAE.

To illustrate the striking differences within CNS-infiltrated $\mathrm{T}$ cells, we generated pie charts showing the proportions of cells with an endogenous (CD45.2) or transferred (CD45.1) origin amongst CD4 (Figure 7C) or CD8 (Figure 7D) T lymphocytes. These representations underline that amongst all
CNS-infiltrating CD4T cells those producing IFN $\gamma$ or GMCSF originated mainly from the transferred cells (Figure 7C). The second and third column of pies also illustrate the greater proportion of transferred CD4 T cells (CD45.1) producing these cytokines. In contrast, in the CD8 T cell compartment, most cells expressing effector molecules (IFN $\gamma$, granzyme B or NKG2D) originated from the endogenous compartment (Figure 7D). In fact, as mentioned above, few transferred CD8 T cells (CD45.1 ${ }^{+}$) reached the CNS although similar amount of these cells were cotransferred with CD4 T cells. Nevertheless, most CD45.1 ${ }^{+}$CD8 T cells that reached the CNS were able to produce IFN $\gamma$ and an important proportion expressed NKG2D (Figure 7D second column of pies). Given that a greater proportion of CD8 T cells express NKG2D than CD4 T cells, our results identify CD8 T cells as potential players within the endogenous $\mathrm{NKG}_{2} \mathrm{D}^{+}$immune cells contributing to disease severity in the adoptive model of EAE.

\section{DISCUSSION}

In the present study, we establish a novel role for the NKG2DNKG2DL interaction in the pathobiology of a mouse model of MS. We demonstrate that the activating receptor NKG2D contributes to the pathogenic properties of $\mathrm{T}$ lymphocytes that are recruited to the CNS upon peripheral activation of encephalitogenic T lymphocytes. We show that one particular murine NKG2DL, MULT1 is elevated in the target organ and enhances effector functions of activated CD8 T lymphocytes.

Although EAE models do not recapitulate the full complexity of MS pathobiology, they provide relevant in vivo models to investigate the contribution of immune factors to different disease stages such as initiation, progression or recovery (31). We investigated the role of NKG2D using these in vivo MS models. We induced active EAE in $\mathrm{Klrk1}^{-/-}$and $\mathrm{Klrk1} \mathrm{I}^{+/+}$ mice from the same breeding colony and noted only a small difference in neurobehavioral scores (Figure 1A). Our results confirm that in the absence of NKG2D, active EAE develops with similar kinetics and amplitude than when this receptor is present. Indeed, Guerra and colleagues observed a slightly reduced EAE disease in $K l r k 1^{-/-}$compared to wild type mice only when using a suboptimal dose of $\mathrm{MOG}_{35-55}$ (19). It is well established that active EAE is strongly driven by CD4 T lymphocytes. However, NKG2D is only expressed on a small proportion of activated CD4 T lymphocytes $(<10 \%$ Figure 1C); thus, we can speculate that NKG2D does not alter the encephalitogenic potential of CD4 T lymphocytes in optimal active disease conditions. Ruck and colleagues showed that inhibiting NKG2D after immunization but prior to disease onset reduced disease; however, blocking NKG2D after disease onset had no impact on EAE (17). These authors provided data supporting the idea that blocking NKG2D reduced the migration of T lymphocytes into the CNS. Their results suggest that if NKG2D is blocked while CD4 T lymphocytes that have been activated in response to the immunization are traveling from the periphery to the CNS, it is possible to dampen their capacity to enter this organ. Blocking NKG2D prior to disease onset 

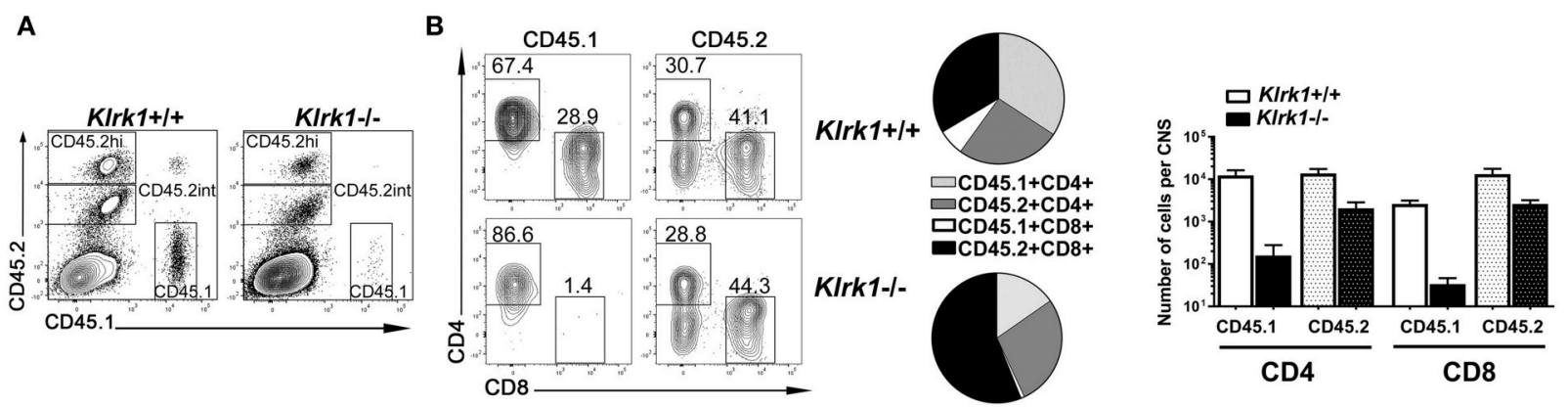

C
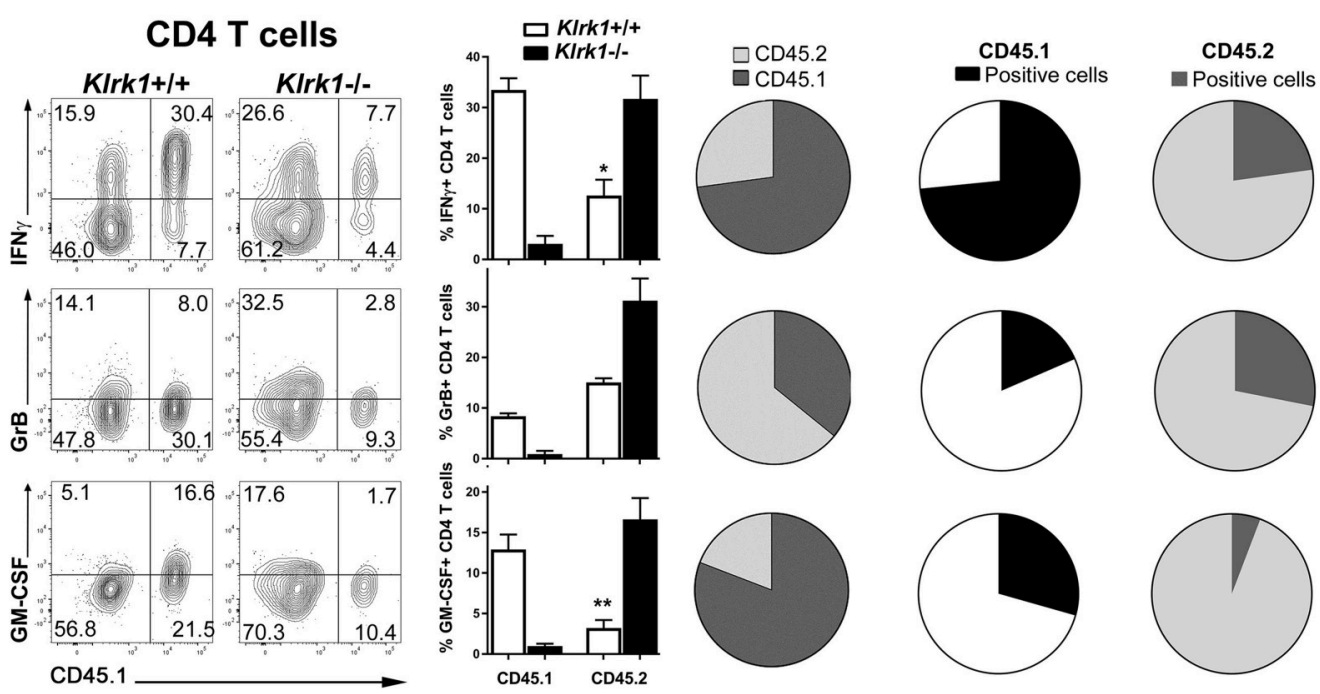

D

CD8 T cells
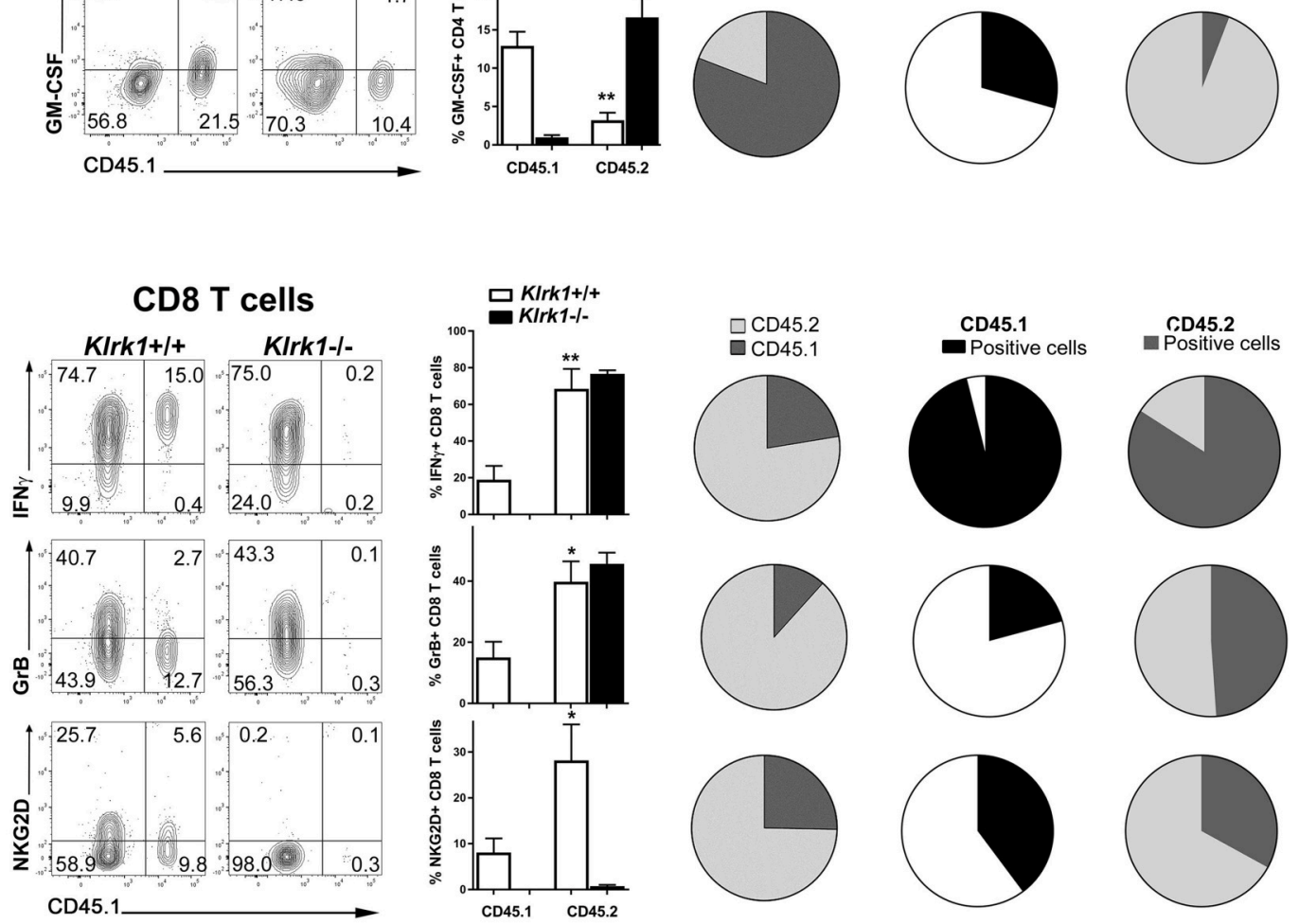

FIGURE 7 | CNS-infiltrated endogenous CD8 lympocytes exhibit effector functions. Leukocytes from MOG-immunized B6-CD45.1 were reactivated in vitro and then adoptively transferred into CD45.2-expressing Klrk1-/- and KIrk $1^{+/+}$mice. Recipients were sacrificed on day 25 and organs collected for flow cytometry analysis. Cells from donor mice were CD45.1 whereas cells from recipients express the standard C57BL/6 CD45.2 marker. Flow data representative of 3-4 mice per group. (A) Representative contour plots of CNS cells in Klrk $1^{-/-}$and KIrk $1^{+/+}$recipient mice showing CD45.1 ${ }^{+}$cells (donor cells) or CD45.2 ${ }^{+}$cells (endogenous cells) amongst all single and living cells. (B) Representative contour plots showing CD45.1+ or CD45.2 ${ }^{+}$gated cells for CD4 and CD8 T cell detection in KIrk1-/- and 


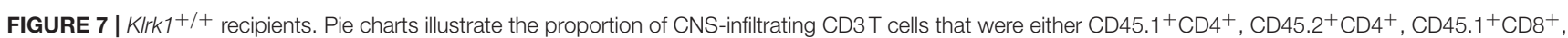
and $\mathrm{CD} 45.2^{+} \mathrm{CD} 8^{+}$in $K / r k 1^{+/+}$and Klrk $1^{-/-}$mice. Number of cells per CNS (spinal cord and brain pooled from each individual mouse) for CD4 and CD8T cells that expressed either CD45.1 or CD45.2. Mean $+\mathrm{SEM} n=3$. (C,D) Representative contour plots of CNS cells gated either on CD4 (C) or CD8 (D) T cells in KIrk1-/- and KIrk $1^{+/+}$recipients showing donor cells $\left(\mathrm{CD} 45.1^{+}\right)$or endogenous cells (CD45.1 negative) producing IFN $\gamma$, granzyme B (GrB) or GM-CSF or expressing NKG2D. Bar graphs illustrate mean \pm SEM $n=3-4$ individual mice of one representative experiment. Statistical differences for percentage of IFN $\gamma$ producing CD4 T cells in Klrk $1+/+$ : CD45.1 in vs. CD45.2 ${ }^{*} p<0.05$; percentage of GM-CSF producing CD4T cells in Klrk ${ }^{+/+}$: CD45.1 in vs. CD45.2 ${ }^{* *} p<0.01$; for percentage of IFN $\gamma$ producing CD8T cells in KIrk1+/+: CD45.1 in vs. CD45.2 ${ }^{* *} p<0.01$; percentage of granzyme B producing CD8T cells in KIrk1+/+: CD45.1 in vs. CD45.2 ${ }^{\star} p<0.05$; percentage of NKG2D-expressing CD8T cells in Klrk $1^{+/+}$: CD45.1 in vs. CD45. ${ }^{*} p<0.05$. All pies show proportion of T cells in Klrk $1^{+/+}$recipients. Pies in first column illustrate percentage of T cells producing IFN $\gamma$, granzyme B (GrB) or GM-CSF or expressing NKG2D according to the expression of CD45.2 (light gray) or CD45.1 (dark gray). Pies in second (CD45.1) and third (CD45.2) columns illustrate percentage of cells producing IFN $\gamma$, granzyme B (GrB) or GM-CSF or expressing.NKG2D within these two subsets.

using soluble NKG2D was more efficient at reducing disease than an anti-NKG2D antibody (17). We can speculate that the soluble NKG2D, in contrast to the anti-NKG2D antibody, could bind to soluble forms of NKG2DL and prevent their effects and accumulation. Overall, these experiments suggest that NKG2D plays a role in CD4 $\mathrm{T}$ cell driven active EAE model mainly at disease onset.

To investigate the contribution of NKG2D at the effector phase of EAE and in the absence of adjuvant, we use the passive EAE model. As a greater proportion of activated CD8 T lymphocytes express NKG2D compared to CD4 counterparts (Figure 1D), we optimized our culture conditions to transfer similar proportions of both CD4 and CD8 T cell subsets into naïve recipients. We ruled out any differences in activated wild type lymphocytes between groups and always injected $K l r k 1^{-/-}$and $K l r k 1^{+/+}$mice in parallel. Disease onset was similar in both groups; however, we reproducibly observed reduced disease severity in $K l r k 1^{-/-}$recipients compared to $K l r k 1^{+/+}$counterparts for the entire examination period (Figure 6A). Using CD45.1 and CD45.2 detection, we could easily discriminate endogenous leukocytes (CD45.2) from the transferred immune cells (CD45.1) (Figure 7). A greater proportion of CNS-infiltrated $\mathrm{T}$ cells originated from the transferred CD4 than CD8 T lymphocytes, regardless of their genotype (Figure 7B). In contrast, endogenous CD8 T lymphocytes were more abundant than the endogenous CD4 T lymphocytes (Figure 7B). This observation is relevant as multiple publications have documented that the number of CD8 T lymphocytes is equal or surpasses the number of CD4 T lymphocytes in MS lesions (32-36). Notably, transferred and endogenous $\mathrm{T}$ lymphocytes could produce immune mediators associated with effector functions (IFN $\gamma$, GM-CSF, Granzyme B) (Figure 7). Our results support the notion that endogenous immune cells contribute to the disease severity triggered by the transfer of MOG-activated T cells, via a NKG2D-dependent mechanism (Figure 6).

It is well established that the expression of each NKG2DL is regulated at multiple steps in a cell type specific manner (5-7). It is thus necessary to assess the presence of these ligands at the protein level. We established that one NKG2DL in particular, MULT1, was significantly elevated in the CNS at the peak of EAE in both $K l r k 1^{-/-}$and $K l r k 1^{+/+}$mice while other ligands (Rae1 and H60) were modestly, or not, altered at the mRNA level (Figure 2A). In line with our results, others also reported elevated
mRNA levels of MULT1 in the spinal cord during EAE (30), but they did not assess protein expression. We employed primers detecting all forms of Rae- 1 and observed small variations for the expression of this group of NKG2DL (Figure 2). Using specific primers for two Rae-1 family members, Rae1d and Raele, Djelloul and colleagues observed elevated mRNA levels in lumbar spinal cord of C57BL/6 mice during EAE (30). Rae-1 was detected at low levels by Western blot in spinal cord of both PLP $_{139-151}$ SJL/J and $\mathrm{MOG}_{35-55}$ C57BL/6 EAE mice (37). We detected Rae-1 family members using a pan-antibody by western blot in different CNS areas but did not observe variations between EAE mice and controls (Figures 2B,C). Overall, our data suggest that the protein expression of Rae-1 family members was not significantly modified in the CNS during EAE but we cannot rule out that the expression of specific Rae-1 members was impacted.

In contrast, we established that the protein expression of MULT1 is elevated in the spinal cord at the peak of active EAE disease. Notably, we detected two bands corresponding to the full length $(50-55 \mathrm{kDa})$ and to the cleaved protein $(25$ $\mathrm{kDa}$ ) (Figure 3) especially in the spinal cord of EAE mice. The shed form of MULT1 was 4-5 times more abundant than the full length form in spinal cord lysates from EAE animals at the peak of disease (Figure 3). Moreover, we detected an elevated amount of the shed form of MULT1 in the CSF of EAE mice especially at disease peak (Figure 4). We observed similar levels of MULT1 in both $K l r k 1^{-/-}$and $K l r k 1^{+/+}$groups suggesting that the expression of MULT1 does not depend on the presence of NKG2D on immune cells. The soluble shed form of MULT1 was also detectable in the CSF of passive EAE mice (Figure 6) supporting the notion that neuroinflammatory responses triggered by $\mathrm{T}$ cells in the absence of adjuvant also caused MULT1 release in the CSF. Raulet's group detected soluble MULT1 in serum of MULT1 ${ }^{+}$tumor bearing mice and established that the $24 \mathrm{kDa}$ form of MULT1 could be shed via matrix metalloproteinase dependent mechanisms (10). We can speculate that a similar mechanism takes place during EAE as multiple matrix metalloproteinases are elevated in the CNS of EAE mice as well as in the brain tissue, serum and CSF of MS patients $(38,39)$. Overall, our results show that protein levels of MULT1 are enhanced in the CNS during EAE and that this ligand is present in a shed form detectable in tissue and CSF.

Multiple lines of evidence have established that amongst all NKG2DL, MULT1 exhibits unique properties. MULT1 displays the highest affinity for NKG2D (9). Multiple groups have 
documented that in mice and humans, shed NKG2DL can act as decoy ligands by preventing NKG2D-expressing immune effector cells from recognizing NKG2DL-bearing target cells, especially in the context of tumor evasion $(40,41)$. In contrast, the Raulet's group has elegantly shown that shed MULT1 enhances NK cell functions and consequently augments tumor rejection in mice (10). Therefore, we investigated whether soluble MULT1 could similarly enhance $\mathrm{T}$ cell functions. In contrast to NK cells, murine $\mathrm{T}$ cells do not express NKG2D unless they are activated. To mimic the in vivo EAE situation, we activated in vitro splenocytes from naïve $\mathrm{Klrk1}^{+/+}$and $\mathrm{Klrk1} \mathrm{I}^{-/-}$ mice using conditions leading to similar proportion (28-35\%) of CD8 T lymphocytes expressing NKG2D in wild type cells (Figure 5A). We added soluble recombinant MULT1, obtained from the same commercial source used by Raulet's group, to the activated splenocytes. We established that the addition of soluble MULT1 upregulated the proportion of CD8 T lymphocytes expressing granzyme B and the cellular expression levels of IFN $\gamma$ (Figure 5). These boosting effects of soluble MULT1 were not observed in splenocytes from $K l r k 1^{-/-}$mice confirming that these effects required NKG2D expression. Our results establish that soluble MULT1 enhances CD8 T cell effector functions via NKG2D. Overall, our in vivo and in vitro data support the notion that endogenous CD8 T cells contribute to passive EAE pathobiology in a NKG2D-dependent manner and these cells increase their effector functions in response to elevated levels of MULT1. Collectively, our results point to the deleterious role of NKG2D and its ligand MULT1 in the pathobiology of a MS mouse model.

The potential contribution of the NKG2D-NKG2DL interaction to the pathobiology of MS in patients is supported by several publications. Wiendl's group observed that CD4 T lymphocytes carrying NKG2D are enriched in the blood, CSF and post-mortem brain lesions of MS patients compared to control donors especially during relapses (17). Elevated levels of soluble MICB but not MICA have been reported in serum of MS patients compared to healthy donors and even more during

\section{REFERENCES}

1. Legroux L, Arbour N. Multiple sclerosis and T lymphocytes: an entangled story. J Neuroimmune Pharmacol. (2015) 10:528-46. doi: 10.1007/s11481-015-9614-0

2. Baecher-Allan C, Kaskow BJ, Weiner HL. Multiple sclerosis: mechanisms and immunotherapy. Neuron (2018) 97:742-68. doi: 10.1016/j.neuron.2018.01.021

3. Lanier LL. NKG2D receptor and its ligands in host defense. Cancer Immunol Res. (2015) 3:575-82. doi: 10.1158/2326-6066.CIR-15-0098

4. Carapito R, Bahram S. Genetics, genomics, and evolutionary biology of NKG2D ligands. Immunol Rev. (2015) 267:88-116. doi: 10.1111/imr.12328

5. Stojanovic A, Correia MP, Cerwenka A. The NKG2D/NKG2DL axis in the crosstalk between lymphoid and myeloid cells in health and disease. Front Immunol. (2018) 9:827. doi: 10.3389/fimmu.2018.00827

6. Raulet DH, Gasser S, Gowen BG, Deng W, Jung H. Regulation of ligands for the NKG2D activating receptor. Annu Rev Immunol. (2013) 31:413-41. doi: 10.1146/annurev-immunol-032712-095951

7. Raulet DH, Marcus A, Coscoy L. Dysregulated cellular functions and cell stress pathways provide critical cues for activating and targeting natural killer relapse compared to remitting phase (15). We have previously shown that human oligodendrocytes both in primary cultures and in post-mortem MS brain tissues express at least one of the NKG2DL (MICA/B) (16). To develop an NKG2DL specific therapy, it will be relevant to investigate the presence of all NKG2DL in MS lesions but also their soluble forms in biological fluids such as CSF and serum. A clinical trial using an antiNKG2D antagonist antibody in inflammatory bowel diseases has provided the proof of concept that the NKG2D-NKG2DL interaction is a valid therapeutic target in human inflammatory diseases (42). Based on our results, we suggest that directing therapies at one specific NKG2DL instead of the receptor could potentially provide a more specific approach.

\section{AUTHOR CONTRIBUTIONS}

LL, AM, CL, GD, and SV: conducted experimental work; NA: designed the study; LL, AM, and NA: analyzed and interpreted the data; NA wrote the manuscript and secured funding.

\section{ACKNOWLEDGMENTS}

This study was supported by an operating grant from the Multiple Sclerosis Society of Canada (MSSOC) (grant number EGID 1584). LL held a studentship from the MSSOC. AM obtained a joint studentship from the MSSOC and the Fonds de Recherche du Québec-Santé (FRQS). CL previously obtained a postdoctoral award from Fondation pour l'Aide à la Recherche sur la Sclérose en Plaques (ARSEP) (France) and the FRQS and currently holds a fellowship from the Canadian Institutes of Health Research. We acknowledge the technical assistance of Diane Beauseigle. We thank the animal facility, the flow cytometry core facility and the microscopy core facil ity of the CRCHUM as well as the laboratory members: Marie-Laure Clénet, Negar Farzamkia, and Florent Lemaitre for their suggestions on the manuscript. cells to transformed and infected cells. Immunol Rev. (2017) 280:93-101. doi: $10.1111 / \mathrm{imr} .12600$

8. Dhar P, Wu JD. NKG2D and its ligands in cancer. Curr Opin Immunol. (2018) 51:55-61. doi: 10.1016/j.coi.2018.02.004

9. Carayannopoulos LN, Naidenko OV, Fremont DH, Yokoyama WM. Cutting edge: murine UL16-binding protein-like transcript 1: a newly described transcript encoding a high-affinity ligand for murine NKG2D. J Immunol. (2002) 169:4079-83. doi: 10.4049/jimmunol.169.8.4079

10. Deng W, Gowen BG, Zhang L, Wang L, Lau S, Iannello A, et al. Antitumor immunity. A shed NKG2D ligand that promotes natural killer cell activation and tumor rejection. Science (2015) 348:136-9. doi: 10.1126/science.1258867

11. Cox ST, Danby R, Hernandez D, Madrigal JA, Saudemont A. Functional characterisation and analysis of the soluble NKG2D ligand repertoire detected in umbilical cord blood plasma. Front Immunol. (2018) 9:1282. doi: 10.3389/fimmu.2018.01282

12. Carapito R, Gottenberg JE, Kotova I, Untrau M, Michel S, Naegely L, et al. A new MHC-linked susceptibility locus for primary Sjogren's syndrome: MICA. Hum Mol Genet. (2017) 26:2565-76. doi: 10.1093/hmg/ddx135

13. Dai Z, Turtle CJ, Booth GC, Riddell SR, Gooley TA, Stevens AM, et al. Normally occurring NKG2D+CD4+ T cells are immunosuppressive and 
inversely correlated with disease activity in juvenile-onset lupus. $J$ Exp Med. (2009) 206:793-805. doi: 10.1084/jem.20081648

14. Hamada S, Caballero-Benitez A, Duran KL, Stevens AM, Spies T, Groh V. Soluble MICB in plasma and urine explains population expansions of NKG2D(+)CD4 T cells inpatients with juvenile-onset systemic lupus erythematosus. Open J Immunol. (2017) 7:1-17. doi: 10.4236/oji.2017.71001

15. Fernandez-Morera JL, Rodriguez-Rodero S, Lahoz C, Tunon A, Astudillo A, Garcia-Suarez O, et al. Soluble MHC class I chain-related protein B serum levels correlate with disease activity in relapsing-remitting multiple sclerosis. Hum Immunol. (2008) 69:235-40. doi: 10.1016/j.humimm.2008.01.021

16. Saikali P, Antel JP, Newcombe J, Chen Z, Freedman M, Blain M, et al. NKG2D-mediated cytotoxicity toward oligodendrocytes suggests a mechanism for tissue injury in multiple sclerosis. J Neurosci. (2007) 27:12208. doi: 10.1523/JNEUROSCI.4402-06.2007

17. Ruck T, Bittner S, Gross CC, Breuer J, Albrecht S, Korr S, et al. CD4+NKG2D+ T cells exhibit enhanced migratory and encephalitogenic properties in neuroinflammation. PLoS ONE (2013) 8:e81455. doi: 10.1371/journal.pone.0081455

18. Constantinescu CS, Farooqi N, O'Brien K, Gran B. Experimental autoimmune encephalomyelitis (EAE) as a model for multiple sclerosis (MS). $\mathrm{Br} J$ Pharmacol. (2011) 164:1079-106. doi: 10.1111/j.1476-5381.2011.01302.x

19. Guerra N, Pestal K, Juarez T, Beck J, Tkach K, Wang L, Raulet DH. A selective role of NKG2D in inflammatory and autoimmune diseases. Clin Immunol. (2013) 149:432-9. doi: 10.1016/j.clim.2013.09.003

20. Legroux L, Pittet CL, Beauseigle D, Deblois G, Prat A, Arbour N. An optimized method to process mouse CNS to simultaneously analyze neural cells and leukocytes by flow cytometry. J Neurosci Methods (2015) 247:23-31. doi: 10.1016/j.jneumeth.2015.03.021

21. Elpek KG, Rubinstein MP, Bellemare-Pelletier A, Goldrath AW, Turley SJ. Mature natural killer cells with phenotypic and functional alterations accumulate upon sustained stimulation with IL-15/IL-15Ralpha complexes. Proc Natl Acad Sci USA. (2010) 107:21647-52. doi: 10.1073/pnas.1012 128107

22. Pittet CL, Newcombe J, Antel JP, Arbour N. The majority of infiltrating CD8 T lymphocytes in multiple sclerosis lesions is insensitive to enhanced PD-L1 levels on CNS cells. Glia (2011) 59:841-56. doi: 10.1002/glia.21158

23. Schneider R, Yaneva T, Beauseigle D, El-Khoury L, Arbour N. IL-27 increases the proliferation and effector functions of human naive CD8+ T lymphocytes and promotes their development into Tc1 cells. Eur J Immunol. (2011) 41:47-59. doi: 10.1002/eji.201040804

24. Gilda JE, Gomes AV. Western blotting using in-gel protein labeling as a normalization control: stain-free technology. Methods Mol Biol. (2015) 1295:381-91. doi: 10.1007/978-1-4939-2550-6_27

25. Arbour N, Holz A, Sipe JC, Naniche D, Romine JS, Zyroff J, et al. A new approach for evaluating antigen-specific $\mathrm{T}$ cell responses to myelin antigens during the course of multiple sclerosis. J Neuroimmunol. (2003) 137:197-209. doi: 10.1016/S0165-5728(03)00080-8

26. Arbour N, Naniche D, Homann D, Davis RJ, Flavell RA, Oldstone MB. c-Jun $\mathrm{NH}(2)$-terminal kinase (JNK)1 and JNK2 signaling pathways have divergent roles in CD8(+) $\mathrm{T}$ cell-mediated antiviral immunity. J Exp Med. (2002) 195:801-10. doi: 10.1084/jem.20011481

27. Malarkannan S, Shih PP, Eden PA, Horng T, Zuberi AR, Christianson G, et al. The molecular and functional characterization of a dominant minor $\mathrm{H}$ antigen, H60. J Immunol. (1998) 161:3501-9.

28. Carayannopoulos LN, Naidenko OV, Kinder J, Ho EL, Fremont DH, Yokoyama WM. Ligands for murine NKG2D display heterogeneous binding behavior. Eur J Immunol. (2002) 32:597-605. doi: 10.1002/15214141(200203)32:3<597::AID-IMMU597>3.0.CO;2-E

29. Lodoen M, Ogasawara K, Hamerman JA, Arase H, Houchins JP, Mocarski ES, et al. NKG2D-mediated natural killer cell protection against cytomegalovirus is impaired by viral gp40 modulation of retinoic acid early inducible 1 gene molecules. J Exp Med. (2003) 197:1245-53. doi: 10.1084/jem.200 21973

30. Djelloul M, Popa N, Pelletier F, Raguenez G, Boucraut J. RAE-1 expression is induced during experimental autoimmune encephalomyelitis and is correlated with microglia cell proliferation. Brain Behav Immun. (2016) 58:209-17. doi: 10.1016/j.bbi.2016.07.147

31. Bjelobaba I, Begovic-Kupresanin V, Pekovic S, Lavrnja I. Animal models of multiple sclerosis: Focus on experimental autoimmune encephalomyelitis. $J$ Neurosci Res. (2018) 96:1021-42 doi: 10.1002/jnr.24224

32. Frischer M, Bramow S, Dal-Bianco A, Lucchinetti CF, Rauschka $\mathrm{H}$, Schmidbauer $\mathrm{M}$, et al. The relation between inflammation and neurodegeneration in multiple sclerosis brains. Brain (2009) 132(Pt 5):1175-89. doi: 10.1093/brain/awp070

33. Junker A, Ivanidze J, Malotka J, Eiglmeier I, Lassmann H, Wekerle H, et al. Multiple sclerosis: T-cell receptor expression in distinct brain regions. Brain (2007) 130(Pt 11):2789-99. doi: 10.1093/brain/awm214

34. Lassmann H, Ransohoff RM. The CD4-Th1 model for multiple sclerosis: a critical [correction of crucial] re-appraisal. Trends Immunol. (2004) 25:132-7 doi: 10.1016/j.it.2004.01.007

35. Neumann H, Medana IM, Bauer J, Lassmann H. Cytotoxic T lymphocytes in autoimmune and degenerative CNS diseases. Trends Neurosci. (2002) 25:313-9. doi: 10.1016/S0166-2236(02)02154-9

36. Machado-Santos J, Saji E, Troscher AR, Paunovic M, Liblau R, Gabriely G, et al. The compartmentalized inflammatory response in the multiple sclerosis brain is composed of tissue-resident CD8+ T lymphocytes and B cells. Brain (2018) 141:2066-82. doi: 10.1093/brain/awy151

37. Galazka G, Jurewicz A, Orlowski W, Stasiolek M, Brosnan CF, Raine $\mathrm{CS}$, et al. EAE tolerance induction with Hsp70-peptide complexes depends on H60 and NKG2D activity. J Immunol. (2007) 179:4503-12. doi: 10.4049/jimmunol.179.7.4503

38. Yong VW, Zabad RK, Agrawal S, Goncalves Dasilva A, Metz LM. Elevation of matrix metalloproteinases (MMPs) in multiple sclerosis and impact of immunomodulators. J Neurol Sci. (2007) 259:79-84. doi: 10.1016/j.jns.2006.11.021

39. Weaver A, Goncalves da Silva A, Nuttall RK, Edwards DR, Shapiro SD, Rivest $\mathrm{S}$, et al. An elevated matrix metalloproteinase (MMP) in an animal model of multiple sclerosis is protective by affecting Th1/Th2 polarization. FASEB J. (2005) 19:1668-70. doi: 10.1096/fj.04-2030fje

40. Narni-Mancinelli E, Vivier E. Shed NKG2D ligand boosts NK cell immunity. Cell Res. (2015) 25:651-2. doi: 10.1038/cr.2015.41

41. Zingoni A, Molfetta R, Fionda C, Soriani A, Paolini R, Cippitelli M, et al. NKG2D and Its Ligands: "One for All, All for One". Front Immunol. (2018) 9:476. doi: 10.3389/fimmu.2018.00476

42. Allez M, Skolnick BE, Wisniewska-Jarosinska M, Petryka R, Overgaard RV. Anti-NKG2D monoclonal antibody (NNC0142-0002) in active Crohn's disease: a randomised controlled trial. Gut (2017) 66:1918-25. doi: 10.1136/gutjnl-2016-311824

Conflict of Interest Statement: The authors declare that the research was conducted in the absence of any commercial or financial relationships that could be construed as a potential conflict of interest.

Copyright (c) 2019 Legroux, Moratalla, Laurent, Deblois, Verstraeten and Arbour. This is an open-access article distributed under the terms of the Creative Commons Attribution License (CC BY). The use, distribution or reproduction in other forums is permitted, provided the original author(s) and the copyright owner(s) are credited and that the original publication in this journal is cited, in accordance with accepted academic practice. No use, distribution or reproduction is permitted which does not comply with these terms. 